The finite field multi-way relay channel with correlated sources: the three-user case

by Lawrence Ong, Roy Timo, Gottfried Lechner, Sarah J. Johnson, \& Christopher M. Kellett

Copyright (C) 2011 IEEE.

This is an author-prepared version of the article, reprinted from IEEE International

Symposium on Information Theory Proceedings, ISIT 2011, p. 2238-2242.

http://dx.doi.org/10.1109/ISIT.2011.6033958

This material is posted here with permission of the IEEE. Such permission of the IEEE does not in any way imply IEEE endorsement of any of University of Newcastle's products or services. Internal or personal use of this material is permitted. However, permission to reprint/republish this material for advertising or promotional purposes or for creating new collective works for resale or redistribution must be obtained from the IEEE by writing to pubs-permissions@ieee.org. By choosing to view this document, you agree to all provisions of the copyright laws protecting it. 


\title{
The Finite Field Multi-Way Relay Channel with Correlated Sources: The Three-User Case
}

\author{
Lawrence Ong ${ }^{\dagger}$, Roy Timo ${ }^{\ddagger}$, Gottfried Lechner ${ }^{\ddagger}$, Sarah J. Johnson ${ }^{\dagger}$, and Christopher M. Kellett ${ }^{\dagger}$ \\ ${ }^{\dagger}$ School of Electrical Engineering and Computer Science, The University of Newcastle, Australia \\ ${ }^{\ddagger}$ Institute for Telecommunications Research, University of South Australia, Australia \\ Email: lawrence.ong@cantab.net, \{roy.timo, gottfried.lechner\}@unisa.edu.au, \{sarah.johnson, chris.kellett\}@newcastle.edu.au
}

\begin{abstract}
The three-user finite field multi-way relay channel with correlated sources is considered. The three users generate possibly correlated messages, and each user is to transmit its message to the two other users reliably in the Shannon sense. As there is no direct link among the users, communication is carried out via a relay, and the link from the users to the relay and those from the relay to the users are finite field adder channels with additive noise of arbitrary distribution. The problem is to determine the set of all possible achievable rates, defined as channel uses per source symbol for reliable communication. For two classes of source/channel combinations, the solution is obtained using Slepian-Wolf source coding combined with functional-decode-forward channel coding.
\end{abstract}

\section{INTRODUCTION}

In this paper we study the three-user finite field multiway relay channel (MWRC) with correlated sources, where each user is to transmit its data to the other two users reliably (in the Shannon sense) via a single relay. For two classes of source/channel combinations, we obtain a complete characterization for reliable communication, i.e., the set of all achievable rates, defined as channel uses per source symbol.

The MWRC is an extension of the two-way relay channel where two users exchange data via a relay [1]-[6]. One application of the MWRC with correlated sources is the communication of weather stations via a satellite, where multiple stations obtain measurements of their respective local weather condition, and each station is to obtain the weather conditions at all other stations by communicating with a satellite.

We study the MWRC in which each user is to decode the data from all other users, and where there is no direct link among the users. Communication is carried out via a single relay. This system has been studied from the point of view of channel coding and source coding, independently, under different setups.

In the channel coding setups, the sources are assumed to be independent, and the channel noisy. The problem formulation is "how many bits of data can each user send per channel use?" Achievable rate tuples here refer to the tuple of the number of message bits (per channel use) the users can transmit such that all other users can reliably recover their messages. The challenge is to find the capacity region which is the closure of all achievable rate tuples. Though the capacity region of the general MWRC remains unknown, Gündüz et al. [7] obtained asymptotic capacity results for the high SNR and the low SNR regimes for the Gaussian MWRC, and Ong et al. [8], [9]

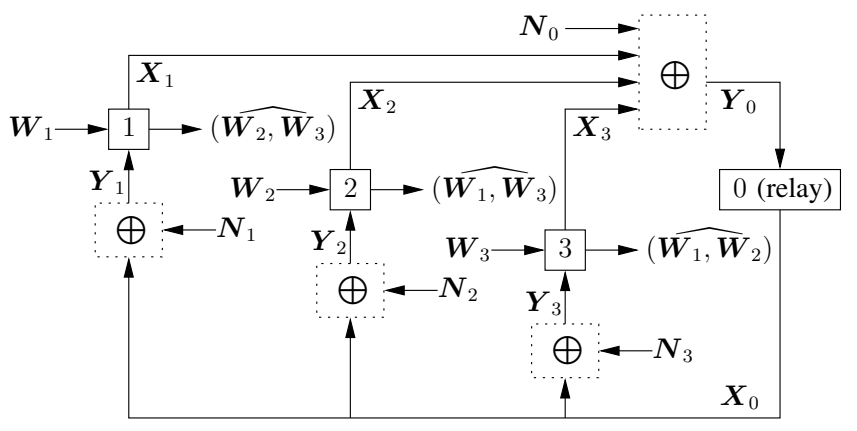

Fig. 1. The three-user finite field MWRC with correlated sources

derived the capacity region of the finite field MWRC, which is achieved by functional-decode-forward channel coding.

In the source coding setups, the sources are assumed to be correlated, but the channel noiseless. The problem formulation is "how many bits does each node need to encode per message symbol?" Achievable rate tuples here refer to the tuple of the number of bits (per source symbol) allocated to the encoders for which reliable reconstruction of each message is possible by all other users. The challenge is to find the set of all achievable rate tuples. The source coding problem for the three-user MWRC was solved by Wyner et al. [10], using cascaded Slepian-Wolf source coding [11].

In this paper, we study both source and channel coding in one setup, i.e., the three-user MWRC with noisy channel and with correlated sources (see our recent work [12] on the two-user MWRC with correlated sources). In the multiterminal network, it is well known that solving the source coding and the channel coding problems separately does not solve the source-channel problem, i.e., noisy channels with correlated sources (see, e.g., the multiple-access channel [13]). We will, however, show that a strategy using Slepian-Wolf source coding (an optimal source coding) and functionaldecode-forward channel coding (an optimal channel coding) is optimal for two classes of the finite field MWRCs with correlated sources. Using an example, we will also show that this scheme might not be optimal in general.

\section{MAin Results}

\section{A. Channel Model}

Referring to Fig. 1, nodes 1, 2, and 3 are the users and node 0 the relay. Let $W_{i}$ be the message of user $i$, and each message 
triplet $\left(W_{1}, W_{2}, W_{3}\right)$ be independently generated according to $p\left(w_{1}, w_{2}, w_{3}\right)$. The memoryless finite field channel is defined by (i) the uplink: $Y_{0}=X_{1} \oplus X_{2} \oplus X_{3} \oplus N_{0}$ and (ii) the downlinks: $Y_{i}=X_{0} \oplus N_{i}$, for $i \in\{1,2,3\}$, where $X_{j}, Y_{j}, N_{j} \in \mathcal{F}, \forall j \in\{0,1,2,3\}$, where $\mathcal{F}$ is a finite field with addition $\oplus, X_{j}$ is the channel input from node $j, Y_{j}$ is the channel output received by node $j$, and $N_{j}$ is the receiver noise of node $j$. The noise terms $N_{j}$ 's are independent for each user and for each channel use. If the users can reliably ${ }^{1}$ exchange $m$ message triplets in $n$ channel uses, the rate of $\kappa=n / m$ channel uses per source symbol is said to be achievable.

\section{B. Definitions}

Before presenting the main results of this paper, we define the following properties for the sources and the channel:

The channel is

- symmetrical, if all the downlinks from the relay to the users are equally noisy, i.e., $H\left(N_{1}\right)=H\left(N_{2}\right)=H\left(N_{3}\right)$;

- asymmetrical, otherwise.

Remark 1: For a symmetrical channel, we do not impose the constraint that the "noise power" at the relay, $H\left(N_{0}\right)$, must equal that at the users. This means that all downlinks from the relay to the users are equally noisy, but the uplink from the users to the relay can be noisier or less noisy.

The sources have

- almost-balanced conditional mutual information (ABCMI), if

$$
\begin{array}{r}
I\left(W_{i} ; W_{j} \mid W_{k}\right) \leq I\left(W_{j} ; W_{k} \mid W_{i}\right)+I\left(W_{i} ; W_{k} \mid W_{j}\right), \\
\forall i, j, k \in\{1,2,3\} \text { and } i \neq j \neq k ;
\end{array}
$$

- unbalanced conditional mutual information, otherwise, i.e., there exists some user $A \in\{1,2,3\}$, such that

$$
\begin{aligned}
I\left(W_{B} ; W_{C} \mid W_{A}\right)=I\left(W_{A} ; W_{B} \mid W_{C}\right) & \\
& +I\left(W_{A} ; W_{C} \mid W_{B}\right)+\eta,
\end{aligned}
$$

for some $\eta>0$ and $B, C \in\{1,2,3\} \backslash\{A\}$ where $B \neq C$. Note that if (1) fails, then there necessarily exists an appropriate $\eta$ as in (2).

For the class of sources with unbalanced conditional mutual information, we say that the sources have skewed conditional entropies (SCE) if in addition to (2), we have the following:

$$
\begin{aligned}
& H\left(W_{B}, W_{C} \mid W_{A}\right) \geq \max \{ H\left(W_{A}, W_{B} \mid W_{C}\right), \\
&\left.H\left(W_{A}, W_{C} \mid W_{B}\right)\right\}+\eta,
\end{aligned}
$$

for the same $\eta$ as in (2).

Fig. 2 shows the relationship among the entropies and mutual information for the three source messages $W_{1}, W_{2}$, and $W_{3}$ for the cases of ABCMI and SCE. Referring to Fig. 2(a), the shaded areas refer to the mutual information between any two source messages given the third source message. For

\footnotetext{
${ }^{1}$ Reliability is defined in the Shannon sense, i.e., the probability that any user wrongly decodes any other user's message can be made as small as desired.
}

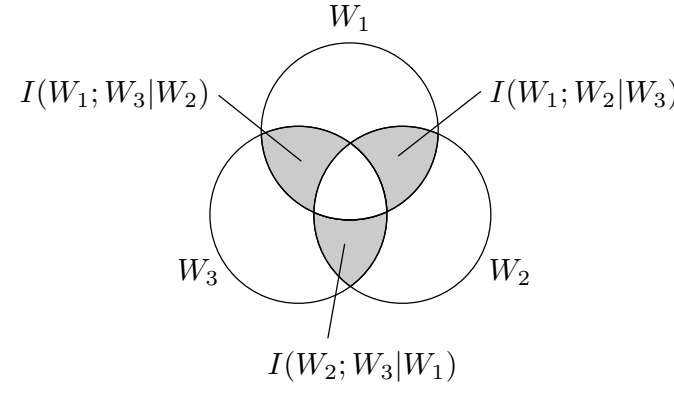

(a) almost-balanced conditional mutual information (ABCMI)

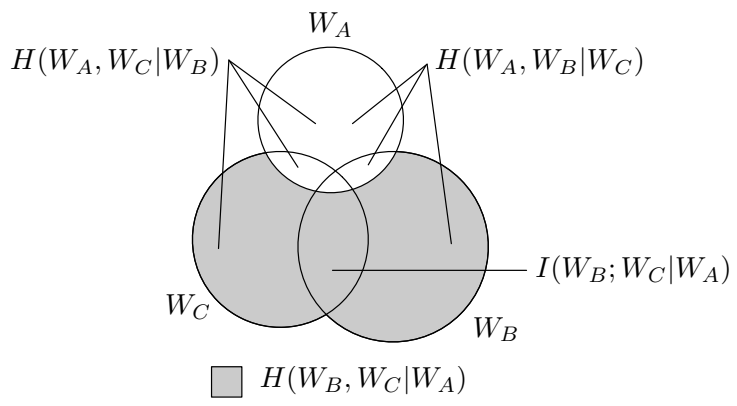

(b) skewed conditional entropies (SCE)

Fig. 2. Information diagrams for the sources

ABCMI, we have that any of the three shaded areas must not be bigger than the sum of the other two shaded areas. Suppose that the sources do not have ABCMI, then they must have unbalanced conditional mutual information, i.e., we can find a user $A$ where $I\left(W_{B} ; W_{C} \mid W_{A}\right)$ is larger than the sum of $I\left(W_{A} ; W_{B} \mid W_{C}\right)$ and $I\left(W_{A} ; W_{C} \mid W_{B}\right)$ by an amount $\eta$ (see Fig. 2(b)). In addition, for sources with SCE, we also have that for the two messages, $W_{B}$ and $W_{C}$, whose mutual information conditioned on $W_{A}$, i.e., $I\left(W_{B} ; W_{C} \mid W_{A}\right)$, is larger than the sum of the other two pairs by the amount $\eta$, their entropy conditioned on $W_{A}$, i.e., $H\left(W_{B}, W_{C} \mid W_{A}\right)$, is also greater than that of any other pair (conditioned on the message of the third user) by at least $\eta$.

\section{Main Results}

In this paper, we show that for the two cases of (i) sources with ABCMI, and (ii) sources with SCE on symmetrical channels, necessary and sufficient conditions for a rate $\kappa>0$ to be achievable are

$$
\begin{aligned}
H\left(W_{j}, W_{k} \mid W_{i}\right) \leq \kappa\left[\log _{2}|\mathcal{F}|-\max \left\{H\left(N_{0}\right), H\left(N_{i}\right)\right\}\right] \\
\forall i, j, k \in\{1,2,3\} \text { and } i \neq j \neq k .
\end{aligned}
$$

For the remaining cases, the above conditions are necessary, but may not be sufficient. For these cases, we obtain sufficient conditions for achievability, but there remains a gap between (4) and the sufficient conditions presented. The results are summarized in Table I.

Remark 2: Utilizing feedback is permitted in our system model. Consider $m$ message triplets, and define $\boldsymbol{W}_{i} \triangleq$ $\left(W_{i}[1], \ldots, W_{i}[m]\right)$. The transmitted channel symbol of 
TABLE I

Main Results: The Set of All Achievable Rates Found for the Following Cases

\begin{tabular}{|c|c|c|c|}
\hline \multirow{2}{*}{ Source structure } & $\begin{array}{c}\text { Almost-balanced conditional } \\
\text { mutual information (ABCMI) }\end{array}$ & \multicolumn{2}{|c|}{ Unbalanced conditional mutual information } \\
\cline { 3 - 4 } & Skewed conditional entropies (SCE) & Others \\
\hline \hline Symmetrical channel & $\sqrt{ }$ & $\sqrt{ }$ & $\times$ \\
Asymmetrical channel & $\sqrt{ }$ & $\times$ & $\times$ \\
\hline
\end{tabular}

node $i$ at any time $t$ is a function of its own messages and its past received channel symbols, i.e, $X_{i}[t]=$ $f_{i, t}\left(\boldsymbol{W}_{i}, Y_{i}[1], \ldots, Y_{i}[t]\right), \forall t \in\{1, \ldots, n\}, \forall i \in\{0,1,2,3\}$, where $\boldsymbol{W}_{0} \triangleq \varnothing$. After $n$ channel uses, each user $i$ then estimates the messages of the other users using its own messages and its $n$ received channel symbols, i.e., $\left(\widehat{\boldsymbol{W}}_{j}, \boldsymbol{W}_{k}\right)=$ $g_{i}\left(\boldsymbol{W}_{i}, Y_{i}[1], \ldots, Y_{i}[n]\right), \forall i, j, k \in\{1,2,3\}$ where $i \neq j \neq k$, and $j<k$.

Now, we will prove the above results in Sections III-IV.

\section{CONDITIONS FOR ACHIEVABILITY}

\section{A. Necessary Conditions for Achievability}

We can show the following necessary conditions for achievability:

Theorem 1: Consider a three-user finite field MWRC with correlated sources. If a rate $\kappa>0$ is achievable then

$$
H\left(W_{j}, W_{k} \mid W_{i}\right) \leq \kappa\left[\log _{2}|\mathcal{F}|-\max \left\{H\left(N_{0}\right), H\left(N_{i}\right)\right\}\right],
$$

for all $i, j, k \in\{1,2,3\}$ where $i \neq j \neq k$.

To prove the above necessary conditions for achievability, we first extend the cut-set argument in [14, pages 587-591] for networks with independent sources to those with correlated sources. We then apply the cut-set argument for networks with correlated sources to the three-user finite field MWRC. The proof is omitted because of space constraints.

\section{B. Sufficient Conditions for Achievability}

We can also show the following sufficient condition for achievability:

Theorem 2: Consider a three-user finite field MWRC with correlated sources. For any $\kappa>0$, if there exist three nonnegative real numbers $R_{1}, R_{2}$, and $R_{3}$ satisfying

$$
\begin{aligned}
R_{i} & \geq \frac{1}{\kappa} H\left(W_{i} \mid W_{j}, W_{k}\right) \\
R_{j}+R_{k} & \geq \frac{1}{\kappa} H\left(W_{j}, W_{k} \mid W_{i}\right) \\
R_{j}+R_{k} & \leq \log _{2}|\mathcal{F}|-\max \left\{H\left(N_{0}\right), H\left(N_{i}\right)\right\},
\end{aligned}
$$

for all $i, j, k \in\{1,2,3\}$ where $i \neq j \neq k$, then the rate $\kappa$ is achievable.

Remark 3: $R_{i}$ in the above theorem is the "rate" (in bits per channel use) of the encoded message at which node $i$ transmits on the uplink, for $i \in\{1,2,3\}$.

Sketch of proof for Theorem 2: To prove the above sufficient condition for achievability, we use the techniques of (i) Slepian-Wolf source coding for the noiseless MWRC with correlated sources [10], and (ii) functional-decode-forward channel coding for the finite field MWRC with independent sources [9]. We first use Slepian-Wolf source coding: each user $i$ encodes its message $\boldsymbol{W}_{i}$ to an $n R_{i}$-bit message $M_{i}$, for $i \in\{1,2,3\}$. We then use functional-decode-forward channel coding on the finite field (noisy) channel: each user $i$ transmits $M_{i}$ using a random linear code; the relay decodes a linear function of messages $\left(M_{1}, M_{2}, M_{3}\right)$, denoted by $\boldsymbol{U}$, and forwards $\boldsymbol{U}$ back to the users. If (8) is satisfied, each user $i$ can decode the encoded messages of the other users, i.e., $M_{j}$ and $M_{k}$. Furthermore, if (6)-(7) are satisfied, each user $i$ can recover $\left(\boldsymbol{W}_{j}, \boldsymbol{W}_{k}\right)$ from $\left(\boldsymbol{W}_{i}, M_{j}, M_{k}\right)$. Note that the source codes and the channel codes can be designed separately and independently. The detail of the proof is omitted because of space constraints.

In the rest of this paper, we denote the above coding strategy of using Slepian-Wolf source coding and functional-decodeforward channel coding by SW-FDF.

Remark 4: SW-FDF proposed here is not simply selecting appropriate channels to support the source coding operation in [10]. In [10], the relay obtains $M_{i}$ from each user $i$ on the uplink, $\forall i$. It then creates random bins for $\left(M_{1}, M_{2}, M_{3}\right)$, and sends the bin index $M_{0}$ to the users. In SW-FDF, we do not merely find channels to support the above, i.e., uplinks that allow the relay to decode $\left(M_{1}, M_{2}, M_{3}\right)$, and downlinks that allow the relay to send $M_{0}$ to the users. In fact, we previously showed that having the relay fully decode $\left(M_{1}, M_{2}, M_{3}\right)$ can be sub-optimal [9]. In SW-FDF, we first use the idea in Slepian-Wolf source coding to generate $\left(M_{1}, M_{2}, M_{3}\right)$, and then use functional-decode-forward for the relay to decode a function of $\left(M_{1}, M_{2}, M_{3}\right)$, which depends on the channel codes chosen, and then forward this function to the users.

Remark 5: Conditions (7)-(8) imply (5) in Theorem 1. In addition, we require (6) for all $i, j, k \in\{1,2,3\}$ where $i \neq j \neq$ $k$, which are source coding constraints, to guarantee reliable communication using SW-FDF.

\section{NECESSARY AND SufFICIENT CONDITIONS}

Now, we find conditions under which the above necessary and sufficient conditions match. Note that any three-user finite field MWRC must belong to one of the following two classes.

\section{A. Sources with Almost-Balanced Conditional Mutual Infor- mation (ABCMI)}

For ABCMI, we have the following constraints:

$$
\begin{array}{r}
I\left(W_{i} ; W_{j} \mid W_{k}\right) \leq I\left(W_{j} ; W_{k} \mid W_{i}\right)+I\left(W_{i} ; W_{k} \mid W_{j}\right), \\
\forall i, j, k \in\{1,2,3\} \text { and } i \neq j \neq k .
\end{array}
$$

We first show the following lemma.

Lemma 1: Consider three (possibly correlated) random variables $W_{1}, W_{2}, W_{3}$, and a positive number $\kappa$. If (9) is 
true, then we can always find three non-negative real numbers $R_{1}, R_{2}, R_{3} \geq 0$, such that

$$
\begin{aligned}
\kappa R_{1} & \geq H\left(W_{1} \mid W_{2}, W_{3}\right) \\
\kappa R_{2} & \geq H\left(W_{2} \mid W_{1}, W_{3}\right) \\
\kappa R_{3} & \geq H\left(W_{3} \mid W_{1}, W_{2}\right) \\
\kappa\left(R_{1}+R_{2}\right) & =H\left(W_{1}, W_{2} \mid W_{3}\right) \\
\kappa\left(R_{1}+R_{3}\right) & =H\left(W_{1}, W_{3} \mid W_{2}\right) \\
\kappa\left(R_{2}+R_{3}\right) & =H\left(W_{2}, W_{3} \mid W_{1}\right) .
\end{aligned}
$$

Proof of Lemma 1: It can be shown that choosing

$$
\begin{aligned}
\kappa R_{i}=H\left(W_{i} \mid W_{j}, W_{k}\right)+\frac{1}{2}\left[I\left(W_{i} ; W_{k} \mid W_{j}\right)\right. \\
\left.+I\left(W_{i} ; W_{j} \mid W_{k}\right)-I\left(W_{j} ; W_{k} \mid W_{i}\right)\right],
\end{aligned}
$$

for $i, j, k \in\{1,2,3\}$ and $i \neq j \neq k$ satisfies (10)-(15).

We now show that for ABCMI, i.e., when (9) is true, the necessary conditions in Theorem 1 are sufficient for reliable communications. Let $\kappa$ be any rate that satisfies (5). First, choosing $R_{1}, R_{2}$, and $R_{3}$ in (16), from Lemma 1, (6)-(7) are satisfied. Substituting (13)-(15) into (5), we get

$$
R_{j}+R_{k} \leq \log _{2}|\mathcal{F}|-\max \left\{H\left(N_{0}\right), H\left(N_{i}\right)\right\},
$$

for all $i, j, k \in\{1,2,3\}$ where $i \neq j \neq k$. This is equivalent to (8). This means the rate $\kappa$ is achievable.

So, we have the following necessary and sufficient conditions for reliable communications for ABCMI:

Theorem 3 (ABCMI): Consider a three-user finite field MWRC with correlated sources. If the sources have ABCMI, then the rate $\kappa$ is achievable if and only if

$$
H\left(W_{j}, W_{k} \mid W_{i}\right) \leq \kappa\left[\log _{2}|\mathcal{F}|-\max \left\{H\left(N_{0}\right), H\left(N_{i}\right)\right\}\right],
$$

for all $i, j, k \in\{1,2,3\}$ where $i \neq j \neq k$.

Remark 6: If the sources are independent, we have $H\left(W_{i}, W_{j} \mid W_{k}\right)=H\left(W_{i}\right)+H\left(W_{j}\right)$ for all $i, j, k \in\{1,2,3\}$, $i \neq j \neq k$. In this case, (9) is always satisfied. So, reliable communication is possible if and only if $r_{j}+r_{k} \leq \log _{2}|\mathcal{F}|-$ $\max \left\{H\left(N_{0}\right), H\left(N_{i}\right)\right\}$, for all $i, j, k \in\{1,2,3\}$ and $i \neq j \neq k$, where $r_{j}=H\left(W_{j}\right) / \kappa=m H\left(W_{j}\right) / n$ is the number of message bits transmitted by user $j$ per channel use. With this we recover the capacity region of the three-user MWRC with independent sources [9].

\section{B. Sources with Unbalanced Conditional Mutual Information}

For sources with unbalanced conditional mutual information, we have the following constraint: there exists some user $A \in\{1,2,3\}$, such that

$I\left(W_{B} ; W_{C} \mid W_{A}\right)=I\left(W_{A} ; W_{B} \mid W_{C}\right)+I\left(W_{A} ; W_{C} \mid W_{B}\right)+\eta$,

for some $\eta>0$, for some $B, C \in\{1,2,3\} \backslash\{A\}$, and $B \neq C$. We can show that if the sources do not have ABCMI (i.e., (9) is false), they must have unbalanced conditional mutual information. Now we prove the following lemma:

Lemma 2: Consider three (possibly correlated) random variables $W_{1}, W_{2}, W_{3}$, and a positive number $\kappa$. If (18) is true, then we can always find three non-negative real numbers $R_{A}, R_{B}, R_{C} \geq 0$, such that

$$
\begin{array}{r}
\kappa R_{A}=H\left(W_{A} \mid W_{B}, W_{C}\right) \\
\kappa R_{B}>H\left(W_{B} \mid W_{A}, W_{C}\right) \\
\kappa R_{C}>H\left(W_{C} \mid W_{A}, W_{B}\right) \\
\kappa\left(R_{A}+R_{B}\right)=H\left(W_{A}, W_{B} \mid W_{C}\right) \\
\kappa\left(R_{A}+R_{C}\right)=H\left(W_{A}, W_{C} \mid W_{B}\right) \\
\kappa\left(R_{B}+R_{C}\right)=H\left(W_{B}, W_{C} \mid W_{A}\right),
\end{array}
$$

for the $\eta>0$ in (18).

Proof of Lemma 2: Constraint (18) implies that

$$
\begin{aligned}
& I\left(W_{B} ; W_{C} \mid W_{A}\right)+I\left(W_{A} ; W_{B} \mid W_{C}\right)-I\left(W_{A} ; W_{C} \mid W_{B}\right) \\
& \quad=2 I\left(W_{A} ; W_{B} \mid W_{C}\right)+\eta>0 \\
& I\left(W_{B} ; W_{C} \mid W_{A}\right)+I\left(W_{A} ; W_{C} \mid W_{B}\right)-I\left(W_{A} ; W_{B} \mid W_{C}\right) \\
& \quad=2 I\left(W_{A} ; W_{C} \mid W_{B}\right)+\eta>0 .
\end{aligned}
$$

Now, we choose

$$
\begin{aligned}
\kappa R_{A}= & H\left(W_{A} \mid W_{B}, W_{C}\right) \\
\kappa R_{B}= & H\left(W_{B} \mid W_{A}, W_{C}\right)+\frac{1}{2}\left[I\left(W_{B} ; W_{C} \mid W_{A}\right)\right. \\
& \left.+I\left(W_{A} ; W_{B} \mid W_{C}\right)-I\left(W_{A} ; W_{C} \mid W_{B}\right)\right] \\
\kappa R_{C}= & H\left(W_{C} \mid W_{A}, W_{B}\right)+\frac{1}{2}\left[I\left(W_{B} ; W_{C} \mid W_{A}\right)\right. \\
& \left.+I\left(W_{A} ; W_{C} \mid W_{B}\right)-I\left(W_{A} ; W_{B} \mid W_{C}\right)\right] .
\end{aligned}
$$

Substituting (25) into (28) and (26) into (29), we have $\kappa R_{B}>H\left(W_{B} \mid W_{A}, W_{C}\right)$ and $\kappa R_{C}>H\left(W_{C} \mid W_{A}, W_{B}\right)$ respectively, i.e., (20)-(21) are satisfied. Summing different pairs of (27)-(29), we have $\kappa\left(R_{A}+R_{B}\right)=H\left(W_{A}, W_{B} \mid W_{C}\right)+\eta$, $\kappa\left(R_{A}+R_{C}\right)=H\left(W_{A}, W_{C} \mid W_{B}\right)+\eta$, and $\kappa\left(R_{B}+R_{C}\right)=$ $H\left(W_{B}, W_{C} \mid W_{A}\right)$, i.e., (22)-(24) are satisfied.

1) Skewed Conditional Entropies (SCE): Now, we consider sources with skewed conditional entropies (SCE), i.e., those with unbalanced conditional mutual information with the following additional constraints:

$$
\begin{aligned}
& H\left(W_{B}, W_{C} \mid W_{A}\right) \geq H\left(W_{A}, W_{C} \mid W_{B}\right)+\eta \\
& H\left(W_{B}, W_{C} \mid W_{A}\right) \geq H\left(W_{A}, W_{B} \mid W_{C}\right)+\eta,
\end{aligned}
$$

which is equivalent to $H\left(W_{B}, W_{C} \mid W_{A}\right) \quad \geq$ $\max \left\{H\left(W_{A}, W_{C} \mid W_{B}\right), H\left(W_{A}, W_{B} \mid W_{C}\right)\right\}+\eta$.

For SCE, we have the following result:

Theorem 4 (SCE): Consider a three-user finite field MWRC with correlated sources. For SCE, i.e.,

$$
I\left(W_{B} ; W_{C} \mid W_{A}\right)=I\left(W_{A} ; W_{B} \mid W_{C}\right)+I\left(W_{A} ; W_{C} \mid W_{B}\right)+\eta,
$$

$$
\begin{aligned}
H\left(W_{B}, W_{C} \mid W_{A}\right) \geq \max \{ & H\left(W_{A}, W_{C} \mid W_{B}\right), \\
& \left.H\left(W_{A}, W_{B} \mid W_{C}\right)\right\}+\eta,
\end{aligned}
$$

for some $A, B, C \in\{1,2,3\}, A \neq B \neq C$, and some $\eta>0$, if the channel is symmetrical (refer to the definition in Sec. II-B), then the rate $\kappa$ is achievable if and only if 


$$
H\left(W_{j}, W_{k} \mid W_{i}\right) \leq \kappa\left[\log _{2}|\mathcal{F}|-\max \left\{H\left(N_{0}\right), H\left(N_{i}\right)\right\}\right],
$$

for all $i, j, k \in\{1,2,3\}$ where $i \neq j \neq k$.

Proof of Theorem 4: From Theorem 1, the rate $\kappa$ is achievable only if (34) is satisfied.

For the symmetrical channel, we can define $H\left(N_{\mathrm{d}}\right) \triangleq$ $H\left(N_{1}\right)=H\left(N_{2}\right)=H\left(N_{3}\right)$, where the subscript "d" denotes downlink. Eqn. (33) for SCE implies that (34) reduces to

$$
H\left(W_{B}, W_{C} \mid W_{A}\right) \leq \kappa\left[\log _{2}|\mathcal{F}|-\max \left\{H\left(N_{0}\right), H\left(N_{\mathrm{d}}\right)\right\}\right] .
$$

We need to show that for all symmetrical MWRCs where the sources have SCE, if (35) is satisfied then the rate $\kappa$ is achievable.

From Lemma 2, we can choose three non-negative real numbers $R_{A}, R_{B}$, and $R_{C}$ such that conditions (6)-(7) in Theorem 2 are satisfied. In addition, for the chosen $R_{A}, R_{B}$, and $R_{C}$, we have

$$
\begin{aligned}
\kappa\left(R_{B}+R_{C}\right) & =H\left(W_{B}, W_{C} \mid W_{A}\right) \\
& \leq \kappa\left[\log _{2}|\mathcal{F}|-\max \left\{H\left(N_{0}\right), H\left(N_{\mathrm{d}}\right)\right\}\right] \\
\kappa\left(R_{A}+R_{B}\right) & =H\left(W_{A}, W_{B} \mid W_{C}\right)+\eta \\
& \leq H\left(W_{B}, W_{C} \mid W_{A}\right) \\
& \leq \kappa\left[\log _{2}|\mathcal{F}|-\max \left\{H\left(N_{0}\right), H\left(N_{\mathrm{d}}\right)\right\}\right] \\
\kappa\left(R_{A}+R_{C}\right) & =H\left(W_{A}, W_{C} \mid W_{B}\right)+\eta \\
& \leq H\left(W_{B}, W_{C} \mid W_{A}\right) \\
& \leq \kappa\left[\log _{2}|\mathcal{F}|-\max \left\{H\left(N_{0}\right), H\left(N_{\mathrm{d}}\right)\right\}\right],
\end{aligned}
$$

where (36) follows from (24) and (35); (37) follows from (22), (33), and (35); and (38) follows from (23), (33), and (35). The above three conditions are equivalent to $(8)$ in Theorem 2 . This means the rate $\kappa$ is achievable.

So, for any symmetrical finite field MWRC with correlated sources with SCE, the rate $\kappa$ is achievable if and only if (34) is satisfied.

2) Without Skewed Conditional Entropies: Finally, consider sources without SCE but with unbalanced conditional mutual information, i.e., (18) with the following additional constraint:

$$
\begin{aligned}
& H\left(W_{B}, W_{C} \mid W_{A}\right)<\max \{ H\left(W_{A}, W_{C} \mid W_{B}\right), \\
&\left.H\left(W_{A}, W_{B} \mid W_{C}\right)\right\}+\eta .
\end{aligned}
$$

For this case, we will show that SW-FDF might not be optimal, i.e., not all rates $\kappa$ that satisfy Theorem 1 are achievable using the coding scheme. We consider the source structure depicted in Fig. 3, i.e., $H\left(W_{A}, W_{B} \mid W_{C}\right)=$ $H\left(W_{A}, W_{C} \mid W_{B}\right)=7$ and $H\left(W_{B}, W_{C} \mid W_{A}\right)=5$, and the channel with the following parameters: $\log _{2}|\mathcal{F}|-H\left(N_{0}\right)=7$, $\log _{2}|\mathcal{F}|-H\left(N_{i}\right)=10, \forall i \in\{1,2,3\}$.

The rate of $\kappa=1$ is necessary for reliable communication (from Theorem 1). If $\kappa=1$ is achievable using SW-FDF, from Theorem 2, we must be able to find three non-negative real numbers $R_{A}, R_{B}$, and $R_{C}$ such that

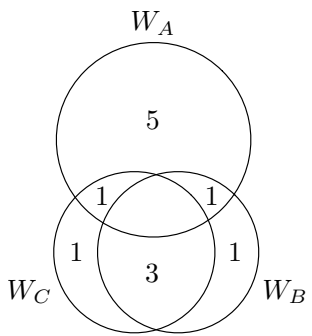

Fig. 3. An example of sources with unbalanced conditional mutual information and without SCE

$$
\begin{aligned}
R_{A} & \geq 5 \\
\min \left\{R_{B}, R_{C}\right\} & \geq 1 \\
\min \left\{R_{A}+R_{B}, R_{A}+R_{C}\right\} & \geq 7 \\
R_{B}+R_{C} & \geq 5 \\
\max \left\{R_{A}+R_{B}, R_{A}+R_{C}, R_{B}+R_{C}\right\} & \leq 7 .
\end{aligned}
$$

From (40) and (43), we must have $\max \left\{R_{A}+R_{B}, R_{A}+\right.$ $\left.R_{C}\right\} \geq 7.5$. This means (44) cannot be satisfied, and hence the rate of $\kappa=1$ is not achievable using SW-FDF.

\section{REFERENCES}

[1] R. Knopp, "Two-way radio networks with a star topology," in Proc. Int. Zurich Semin. Commun. (IZS), Zurich, Switzerland, Feb. 22-24 2006, pp. 154-157.

[2] B. Rankov and A. Wittneben, "Achievable rate regions for the two-way relay channel," in Proc. IEEE Int. Symp. Inf. Theory (ISIT), Seattle, USA, July 9-14 2006, pp. 1668-1672.

[3] — , "Spectral efficient protocols for half-duplex fading relay channels," IEEE J. Sel. Areas Commun., vol. 25, no. 2, pp. 379-389, Feb. 2007.

[4] S. Katti, S. Gollakota, and D. Katabi, "Embracing wireless interference: Analog network coding," in Proc. 2007 ACM SIGCOMM Conf., Kyoto, Japan, Aug. 27-31 2007, pp. 397-408.

[5] D. Gündüz, E. Tuncel, and J. Nayak, "Rate regions for the separated two-way relay channel," in Proc. 46th Allerton Conf. Commun. Control Comput. (Allerton Conf.), Monticello, USA, Sept. 23-26 2008, pp. 1333-1340.

[6] C. Schnurr, S. Stanczak, and T. J. Oechtering, "Achievable rates for the restricted half-duplex two-way relay channel under a partial-decode-andforward protocol," in Proc. IEEE Inf. Theory Workshop (ITW), Porto, Portugal, May 5-9 2008, pp. 134-138.

[7] D. Gündüz, A. Yener, A. Goldsmith, and H. V. Poor, "The multi-way relay channel," in Proc. IEEE Int. Symp. Inf. Theory (ISIT), Seoul, Korea, June 28-July 3 2009, pp. 339-343.

[8] L. Ong, S. J. Johnson, and C. M. Kellett, "An optimal coding strategy for the binary multi-way relay channel," IEEE Commun. Lett., vol. 14, no. 4, pp. 330-332, Apr. 2010.

[9] — "The capacity region of multiway relay channels over finite fields with full data exchange," IEEE Trans. Inf. Theory: Special Issue on Interference Networks, vol. 57, no. 5, pp. 3016-3031, May 2011.

[10] A. D. Wyner, J. K. Wolf, and F. M. J. Willems, "Communicating via a processing broadcast satellite," IEEE Trans. Inf. Theory, vol. 48, no. 6, pp. 1243-1249, June 2002.

[11] D. Slepian and J. K. Wolf, "Noiseless coding of correlated information sources," IEEE Trans. Inf. Theory, vol. IT-19, no. 4, pp. 471-480, July 1973.

[12] R. Timo, L. Ong, and G. Lechner, "The two-way relay network with arbitrarily correlated sources and an orthogonal MAC," in Proc. Data Compression Conf. (DCC), Snowbird, USA, Mar. 29-31 2011, pp. 253262.

[13] G. Dueck, "A note on the multiple access channel with correlated sources," IEEE Trans. Inf. Theory, vol. 27, no. 2, pp. 232-235, Mar. 1981.

[14] T. M. Cover and J. A. Thomas, Elements of Information Theory, 2nd ed. Wiley-Interscience, 2006. 